[2] Denes, J. Keedwell, A. D., Latin squares and their applications. Academic Press, London, 1974.

[3] Panayiotopoulos, J-C., About Latin squares of standard form and of even order. University of Athens, 1976.

Vatazi 16 , Athens 708 , Greece.

Received October, 1976.

The paper appears in Bulletin de la Société Mathématique de Grèce.

\title{
The spectrum of idempotent varieties of algebras with binary operators based on two variable identities
}

N. S. Mendelsohn

The paper contains a proof of the following theorem.

THEOREM. Suppose $V$ is a variety of idempotent algebras with binary operators only and based on two-variable identities and which contains a finite algebra of order $k>1$. Then there exist integers $d, a_{1}, a_{2}, \ldots, a_{r}$ such that if $v$ is the order of a finite algebra in the variety then $v \equiv a_{1}$ or $v \equiv a_{2}$, or $\cdots v \equiv a_{r}(\bmod d)$. Furthermore, there is an integer $v_{0}$ such that if $v \geq v_{0}$ and $v \equiv a_{1}$, or $v \equiv a_{2}, \ldots$, or $v \equiv a_{r}(\bmod d)$ then there is an algebra of order $v$ in the variety.

The theorem has applications to combinatorial designs such as orthogonal latin squares, perpendicular Steiner systems etc. Other applications are to universal algebra itself such as an immediate corollary that Boolean algebras can not be defined by identities using two-variables only.

Received July 6, 1976.

\section{Converse theorems for the trapezoidal rule}

Helmut Brass

Let

$R_{n}[f]=\int_{0}^{1} f(x) \mathrm{d} x-n^{-1}\left\{\frac{1}{2} f(0)+\sum_{\nu=1}^{n-1} f\left(\nu n^{-1}\right)+\frac{1}{2} f(1)\right\}$ 